\title{
Marchiafava Bignami Disease; an uncommon cause of confusion in a long-term alcohol abuser
}

\author{
Amarawardena WKMG ${ }^{1}$, WijesundereAnula ${ }^{2}$, Muhandirum WMT \\ Sri Jayawardenepura General Hospital, Kotte, Sri Lanka
}

Correspondence: Dr. W.K.M.G. Amarawardena

\section{Introduction}

Marchiafava-Bignami Disease (MBD) is a rare demyelinating disorder, mainly affecting the corpus callosum. Following its first description by two Italian pathologists; Ettore Machiafava and Amico Bignami only around 250 patients have been reported in the world literature (1). We present a case of MBD in a 61year old man with chronic alcoholism who presented with neurological symptoms.

\section{Case Report}

A 61 year male with the history of alcoholic liver cell disease was admitted to our ward in a confused state. He had consumed alcohol about 35 units per week for the last 15 years. On admission he was confused, ataxic and talked excessively. He did not have fever, fits, head trauma or inverted sleep pattern. He had truncal ataxia without other clinical features of cerebellar disease. There was no external ophthalmoplegia and fundi were normal. He had ideomotorapraxia with poor attention span. His short term and long term memory and orientation remained intact. The neck was supple. He was mildly icteric and showed episodic flapping tremor.

Initial investigations including full blood count, urinalysis, renal function tests, blood sugar, CRP, serum electrolytes, calcium and magnesium were normal. AST-57u/1, ALT-103u/l. Gamma GT-200u/1. Blood and urine cultures were negative while CSF analysis was normal. Non-contrast CT brain excluded the possibility of chronic subdural haemorrhage. Patient was treated with standard liver failure regimen and intravenous L-Ornithine L-Aspartate for five days, but failed to show any improvement in neurological symptoms and signs.
The possibility of Wernicke's encephalopathy, Korsakoff syndrome or pontinemyelinolysis was considered as the cause of unresolving neurological symptoms in chronic alcohol abuse (2). His cranial MRI showed diffuse high T1/ FLAIR signals in the corpus callosum, appearance suggestive of Marchiafava Bignami disease (Figures 1,2). The definitive diagnosis of MBD, however, requires brain biopsy to demonstrate the degeneration of the corpus callosum. Given the invasive nature of the investigation, high cost and poor prognosis of the illness we did not precede with histological confirmation. He was treated with high dose intravenous thiamine, folic acid, vitamin B12 and other vitamin B supplements. However, neurological symptoms showed only a minimal improvement.

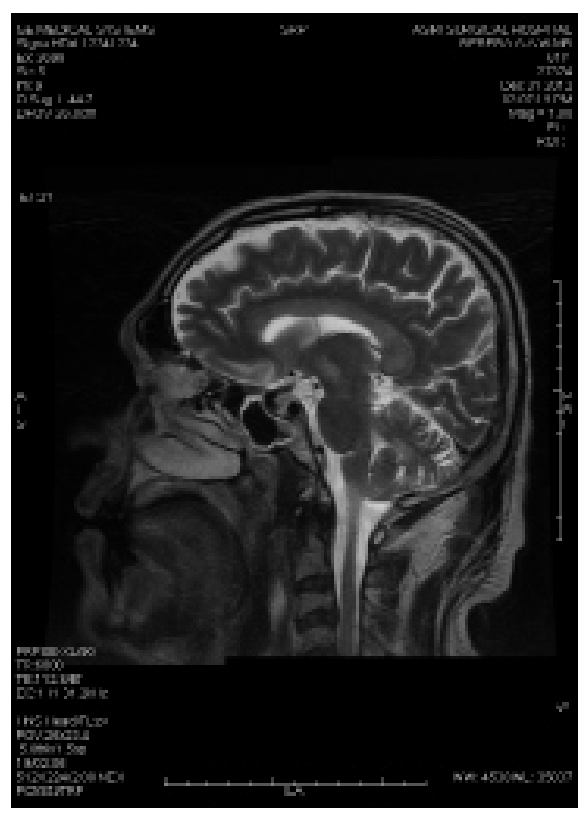

Figure 1: diffuse high T1/ FLAIR signals noted in the corpus callosum-Sagittal view 


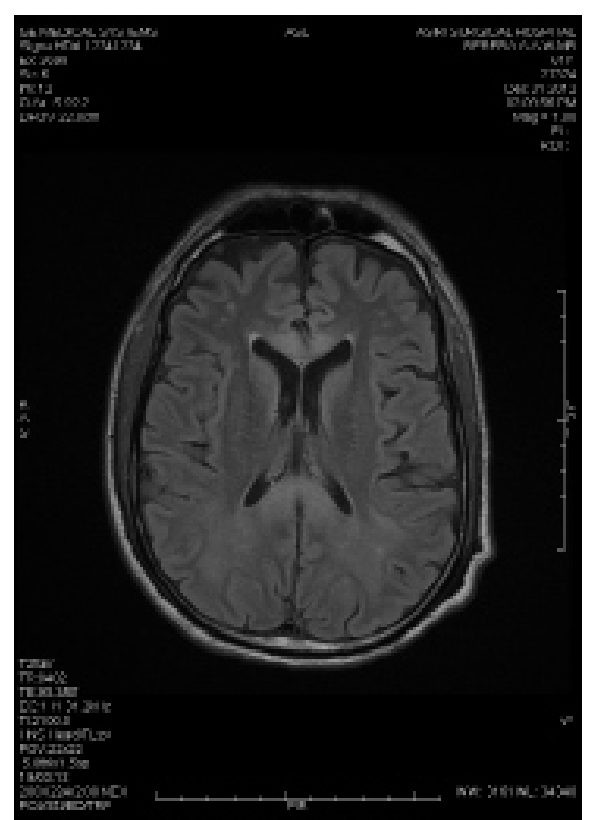

Figure 2: diffuse high T1/ FLAIR signals noted in the corpus callosum-Coronal view

\section{Discussion}

The Italian pathologists Marchiafava and Bignami first described this disease following post-mortem brain analysis of three alcoholics, who presented with status epilepticus and coma in 1903. However, it is felt that MBD goes under-reported and underdiagnosed and that its incidence is probably higher than that reported. This disease is due to the demyelination of the corpus callosum and it can be easily detected with MRI imaging. Presenting features of MBD are nonspecific and include confusion, disorientation, psychotic and emotional symptoms, seizures, hemiparesis, dysarthria, ataxia, coma and death (3). The disorder can be divided into two clinical subtypes. The more severe type (Type A) presents with disorder of consciousness, which may lead to coma and eventually death. The second subtype (Type B) has milder symptoms such as irritability and impairment of gait (4). Apathy, violent tendencies, inappropriate sexual demands, dysarthria, apraxia, hemiparesis and aphasia may coexist. While no specific treatment is available, most patients receive thiamin, folate and other $\mathrm{B}$ vitamins. The effectiveness of such treatment, however, is doubtful. Furthermore, high dose IV thiamine, IV methyl prednisolone and amantadine have been used with varying success (5). Type A has a long term disability of $86 \%$ and a mortality rate of $21 \%$, Type B has a long term disability of $19 \%$ with no excess mortality rate. Based on the clinical information our patient can be categorized to type $\mathrm{B}$ disease (5).

Long-term alcohol abusers presenting with neuropsychiatric manifestations is not an uncommon clinical presentation in our medical wards and many conditions associated with chronic alcohol abuse are considered to explain the clinical presentation. MBD, however, is not a common condition included in this differential diagnosis. The diagnosis can be made on the basis of chronic alcohol abuse associated with unresolving neurological symptoms and MRI findings. We suggest that it is important to consider MBD when nonspecific, non-resolving neurological signs and symptoms persist in an alcoholic despite active treatment for common conditions. Early imaging with MRI should be considered in these cases.

\section{Acknowledgement}

Dr. (Mrs.) P.S.H. Hettiarachchi, Consultant Radiologist, Asiri Surgical Hospital.

\section{References}

1. Shintani $\mathrm{S}$ and Shigaii T. Marchiafava Bignami disease with only slowly progressive cognitive impairment. Journal of Rural Medicine 2007; 1: 62-6.

2. Hlaihel C, Gonnaud PM, Champin S, Rousset H, TranMinh VA, Cotton F. Diffusion-weighted magnetic resonance imaging in Marchiafava-Bignami disease: follow-up studies. Neuroradiology 2005; 47(7): 520-4.

3. Arbelaez A, Pajon A, Castillo M. Acute MarchiafavaBignami disease: MR findings in two patients. American Journal Neuroradiology 2003; 24(10): 1955-7.

4. Heinrich A, Runge U, Khaw AV. Clinicoradiologic subtypes of Marchiafava-Bignami disease. Journal of Neurology 2004; 251:1050-9.

5. Jennifer Ault. Marchiafava-Bignami Disease. Medscape [Internet]. [Updated Feb 3 2012] Available from: http://emedicine.medscape.com/article/1146086overview. 